Research Paper

\title{
Laboratory Parameters are Possible Prognostic Markers in Patients with Advanced-stage NSCLC Treated with Bevacizumab plus Chemotherapy
}

Martin Svaton ${ }^{1 凶}$, Jiri Blazek¹, Gabriela Krakorova ${ }^{1}$, Marcela Buresova1 ${ }^{1}$, Zuzana Teufelova ${ }^{1}$, Josef Vodicka ${ }^{2}$, Karolina Hurdalkova ${ }^{3}$, Magda Barinova ${ }^{3}$ and Milos Pesek ${ }^{1}$

1. Department of Pneumology and Phthisiology, University hospital in Pilsen, Charles University, Faculty of Medicine in Pilsen, Pilsen, Czech Republic

2. Department of Surgery, Charles University, Faculty of Medicine in Pilsen, Pilsen, Czech Republic

3. Institute of Biostatistics and Analyses, Ltd. Brno, Czech Republic

$\square$ Corresponding author: Martin Svaton, Department of Pneumology and Phthisiology, Charles University, Faculty of Medicine in Pilsen, Husova 3, 301 00, Pilsen, Czech Republic. Tel: +420 377402973, E-mail: svatonm@fnplzen.cz

(c) The author(s). This is an open access article distributed under the terms of the Creative Commons Attribution License (https://creativecommons.org/licenses/by/4.0/). See http://ivyspring.com/terms for full terms and conditions.

Received: 2021.01.30; Accepted: 2021.05.29; Published: 2021.07.30

\begin{abstract}
Purpose: To investigate potential associations between selected laboratory markers (CRP, LDH, albumin, sodium, hemoglobin, neutrophils, and neutrophils/lymphocytes ratio [NLR]) and outcomes in patients with non-small cell lung cancer (NSCLC) treated with bevacizumab (BEV) plus chemotherapy.

Patients and Methods: We retrospectively analyzed 105 patients with NSCLC from the Czech TULUNG registry treated at University Hospital in Pilsen with BEV + chemotherapy. Response to therapy was tested by Fisher's exact test. Survival statistics were evaluated using the Kaplan-Meier method and Cox analysis.

Results: We showed significantly better disease control rate when CRP, albumin, hemoglobin, and NLR were within established "normal" values. In univariate analysis, normal values of CRP, LDH, albumin, sodium, hemoglobin, neutrophils, and NLR were associated with better overall survival (OS). Normal values of CRP, albumin, hemoglobin, neutrophils, and NLR were associated also with better progression-free survival (PFS). In a multivariate Cox model, normal values of LDH, albumin, and NLR were associated with significantly better OS while normal CRP, albumin, and NLR were associated with better PFS.

Conclusions: LDH and sodium appear to be possible prognostic markers for BEV treatment in combination with chemotherapy in NSCLC. The parameters associated with inflammatory response (CRP, NLR, albumin, and possibly hemoglobin) appear to be promising predictive markers for this treatment combination.
\end{abstract}

Key words: bevacizumab, inflammation, CRP, albumin, NSCLC, predictive, prognostic

\section{Introduction}

Bevacizumab (BEV) is an intravenously administered monoclonal antibody targeting vascular endothelial growth factor (VEGF) that is widely used in treating patients with advanced non-small cell lung cancer (NSCLC) [1]. The Eastern Cooperative Oncology Group (ECOG) 4599 phase III trial showed a significant survival benefit from using BEV in combination with carboplatin and paclitaxel $(\mathrm{CP})$ in comparison with $\mathrm{CP}$ chemotherapy alone in patients with previously untreated advanced, metastatic, or recurrent NSCLC [2]. Such results were achieved using BEV as maintenance therapy until disease progression. It has been demonstrated previously that the superiority of BEV is limited to patients with non-squamous histology due to higher proportion of potentially risky hemoptysis in squamous lung cancers [3]. Aside from the non-squamous histology, there remains to date no molecular biomarker 
available for predicting treatment efficacy of bevacizumab-based therapy.

A number of past studies have sought an effective predictive marker for this treatment [4-7]. In particular, there have been attempts to use the expression of VEGF, the effect of arterial hypertension, or examination by perfusion computed tomography (CT) to determine the effect of angiogenesis in a given tumor [4-6]. None of these, however, have been found sufficiently reliable or subsequently verified satisfactorily by prospective work to be put into routine clinical practice [3].

Laboratory parameters have been shown to be potential predictors of treatment in a number of other studies involving NSCLC [8-11]. Moreover, some laboratory parameters (mainly C-reactive protein $[\mathrm{CRP}]$, lactate dehydrogenase [LDH], and neutrophils/lymphocytes ratio [NLR]) have shown potential as prognostic or even predictive markers of bevacizumab-based therapy in tumors relating to colorectal cancer, breast cancer, pancreatic cancer, renal cell cancer, glioblastoma, and ovarian cancer [12-17]. This topic has not been comprehensively studied, however, for the combination of chemotherapy and bevacizumab in NSCLC. These laboratory findings may be important decision-making inputs prior to initiating various immunotherapy options, including that of the chemotherapy + bevacizumab combination providing a therapeutic basis for further combination with atezolizumab.

Given this background, the aim of the present study was to investigate potential associations between selected laboratory markers and outcomes in NSCLC patients treated with BEV plus chemotherapy.

\section{Patients and Methods}

\section{Study design and treatment}

Clinical data of patients with cytologically or histologically confirmed advanced NSCLC treated with BEV and chemotherapy (mainly $\mathrm{CP}$ ) were analyzed retrospectively. The patients were treated in the first, or rarely in the second, line of treatment at the Department of Pneumology and Phthisiology, University Hospital in Pilsen in the Czech Republic between 2010 and 2020. BEV was administered intravenously at the approved dose of $7.5 \mathrm{mg} / \mathrm{kg}$ every 3 weeks together with standard platinum doublet chemotherapy. The BEV treatment was administered until progression or unacceptable toxicity, and chemotherapy was given to 4 cycles. Clinical follow-up including physical examination, chest X-ray, and routine laboratory tests was made at least every 4 weeks. CT or positron-emission tomography (PET)/CT were performed at regular intervals according to the local standards or, when progression was suspected, based on clinical or chest X-ray examination. Laboratory markers investigated in the present study included CRP, LDH, albumin, sodium $(\mathrm{Na})$, calcium $(\mathrm{Ca})$, hemoglobin $(\mathrm{Hb})$, neutrophils (Neu), lymphocytes, and NLR. These markers were measured at the initiation of bevacizumab treatment. Serving as the data source was TULUNG, a national non-interventional post-registration database of epidemiological and clinical data for patients with advanced-stage NSCLC treated with targeted or biological therapies in the Czech Republic. We used data recorded from our center (University Hospital in Pilsen) relating to all of our patients in the register who had been treated with chemotherapy and bevacizumab. The patients had given their informed consent to be included into this database and for use of these data for scientific purposes.

\section{Statistical methods}

Standard frequency tables and descriptive statistics were used to characterize the sample data set. The overall response rate (ORR) was defined as the best response according to the Response Evaluation Criteria in Solid Tumors (RECIST 1.1) [18]. We compared disease control rates between selected groups. Continuous parameters are described herein using mean with $95 \%$ confidence interval (CI) and median with minimum and maximum, together with the total number of non-missing observations. Categorical parameters were summarized using absolute and relative frequencies. Relative frequencies were calculated based on the number of patients in a relevant subgroup. ORR (difference in complete response + partial response + stable disease between two categories [i.e., parameter within normal range and outside the norm]) was tested by Fisher's exact test. Overall survival (OS) was defined as the time from initiating treatment to the date of death due to any cause. Progression-free survival (PFS) was defined as the time from treatment initiation to the date of first documented progression or death due to any cause. OS and PFS were estimated using the Kaplan-Meier method, and all point estimates include 95\% confidence intervals $(95 \%$ CI $)$. Differences between OS and PFS were tested by log-rank test. Finally, a multivariate Cox proportional hazards model was used to evaluate the relationship of all potential prognostic factors to the survival measures. The cutoff for deciding on statistical significance was set at $\mathrm{a}=0.05$.

The cutoff for laboratory parameters was 
"normal level" versus "abnormal value" (i.e., $>8 \mathrm{mg} / 1$ for CRP, $>4.2 \mu \mathrm{kat} / 1$ for $\mathrm{LDH},<35 \mathrm{~g} / 1$ for albumin, $<137 \mathrm{mmol} / 1$ for $\mathrm{Na},<2.1$ or $>2.6 \mathrm{mmol} / 1$ for $\mathrm{Ca},<135$ $\mathrm{g} / 1$ for men and $<120 \mathrm{~g} / 1$ for women for $\mathrm{Hb},>7 \times 10^{9} / 1$ for Neu, and $<0.8 \times 10^{9} / 1$ for lymphocytes). Cutoff values are based on standardized lower / upper limits from our certified hematological and biochemical laboratory. The cutoff for NLR was set at the median of reference NLR values (i.e., 3.3101).

Statistical analyses were performed using IBM SPSS, Statistics (version 25.0), and R software (version 3.5.1).

Table 1. Baseline patient characteristics.

\begin{tabular}{|c|c|c|}
\hline Parameter & Category & $\mathrm{n}(\%)$ \\
\hline \multirow[t]{2}{*}{ Sex } & Male & $65(61.9 \%)$ \\
\hline & Female & $40(38.1 \%)$ \\
\hline \multirow[t]{3}{*}{ Smoking status } & Non-smoker & $22(21.0 \%)$ \\
\hline & Former smoker & $31(29.5 \%)$ \\
\hline & Smoker & $52(49.5 \%)$ \\
\hline \multirow[t]{3}{*}{ ECOG PS } & 0 & $4(3.8 \%)$ \\
\hline & 1 & $93(88.6 \%)$ \\
\hline & 2 & $8(7.6 \%)$ \\
\hline \multirow[t]{2}{*}{ Line of therapy } & First & $104(99.1 \%)$ \\
\hline & Second & $1(1.0 \%)$ \\
\hline \multirow[t]{2}{*}{ Histology } & Adenocarcinoma & $100(95.2 \%)$ \\
\hline & Other & $5(4.8 \%)$ \\
\hline \multirow[t]{9}{*}{ T (TNM classification) } & $\mathrm{TX}$ & $4(3.8 \%)$ \\
\hline & T1 (valid until 1. 1. 2011) & $3(2.9 \%)$ \\
\hline & $\mathrm{T} 1 \mathrm{a}$ & $1(1.0 \%)$ \\
\hline & $\mathrm{T} 1 \mathrm{~b}$ & $8(7.6 \%)$ \\
\hline & T2 (valid until 1. 1. 2011) & $6(5.7 \%)$ \\
\hline & $\mathrm{T} 2 \mathrm{a}$ & $11(10.5 \%)$ \\
\hline & $\mathrm{T} 2 \mathrm{~b}$ & $7(6.7 \%)$ \\
\hline & T3 & $19(18.1 \%)$ \\
\hline & $\mathrm{T} 4$ & $46(43.8 \%)$ \\
\hline \multirow[t]{5}{*}{ N (TNM classification) } & NX & $5(4.8 \%)$ \\
\hline & N0 & $23(21.9 \%)$ \\
\hline & N1 & $9(8.6 \%)$ \\
\hline & N2 & $23(21.9 \%)$ \\
\hline & N3 & $45(42.9 \%)$ \\
\hline \multirow[t]{6}{*}{ M (TNM classification) } & $\mathrm{MX}$ & $1(1.0 \%)$ \\
\hline & M0 & $12(11.4 \%)$ \\
\hline & M1 (valid until 1. 1. 2011) & $17(16.2 \%)$ \\
\hline & M1a & $27(25.7 \%)$ \\
\hline & M1b & $44(41.9 \%)$ \\
\hline & M1c (valid from TNM8) & $4(3.8 \%)$ \\
\hline \multirow[t]{2}{*}{ Stage } & III & $6(5.8 \%)$ \\
\hline & IV & $99(94.2 \%)$ \\
\hline \multirow[t]{3}{*}{ Type of chemotherapy } & Carboplatin + paclitaxel & $96(91.4 \%)$ \\
\hline & Carboplatin + docetaxel & $2(1.9 \%)$ \\
\hline & Other & $7(6.7 \%)$ \\
\hline
\end{tabular}

ECOG PS: Eastern Cooperative Oncology Group performance status. TNM classification is shown depending upon the dates of patients' inclusion. TNM classification at those times were in accordance with the 7 th or 8 th edition.

\section{Results}

\section{Patient characteristics}

Included into the retrospective analysis were 105 patients, consisting of 65 males and 40 females, with median age of 63 years. The baseline patient characteristics are summarized in Table 1. Due to the low numbers of values outside the normal limits for $\mathrm{Ca}$ and lymphocytes, these laboratory parameters could not be further included into the calculated analyses.

\section{Overall response rate (ORR)}

We showed a significant relationship between ORR (difference in complete response + partial response + stable disease between two categories) and $\mathrm{CRP}$, albumin, $\mathrm{Hb}$, and NLR, as well as a trend in ORR relative to $\mathrm{Neu}(p=0.058)$. Values of these laboratory parameters outside the established standards were associated with poorer disease control rate. All results are summarized in Table 2.

Table 2. Relationships between laboratory parameters and ORR.

\begin{tabular}{llll}
\hline Parameter & Normal & Outside the norm & p-value \\
\hline CRP & $33(86.8 \%)$ & $36(60.0 \%)$ & $\mathbf{0 . 0 0 6}$ \\
LDH & $45(75.0 \%)$ & $24(63.2 \%)$ & 0.258 \\
Albumin & $63(79.7 \%)$ & $6(31.6 \%)$ & $<0.001$ \\
Sodium & $59(71.1 \%)$ & $10(66.7 \%)$ & 0.763 \\
Hemoglobin & $54(81.8 \%)$ & $15(46.9 \%)$ & $<\mathbf{0 . 0 0 1}$ \\
Neutrophils & $50(76.9 \%)$ & $18(56.3 \%)$ & 0.058 \\
NLR & $39(84.8 \%)$ & $29(56.9 \%)$ & $\mathbf{0 . 0 0 4}$
\end{tabular}

CRP: C-reactive protein; LDH: lactate dehydrogenase; NLR:

neutrophil/lymphocyte ratio

\section{Univariate analysis of PFS and OS}

We observed significantly better OS when CRP, $\mathrm{LDH}$, albumin, $\mathrm{Na}, \mathrm{Hb}, \mathrm{Neu}$, and NLR were within normal and significantly better PFS when CRP, albumin, $\mathrm{Hb}$, Neu, and NLR were normal. Values of these laboratory parameters outside the established standards were associated with poorer PFS and/or OS. These results are summarized in Table 3. KaplanMeier curves for PFS and OS of significant parameters are shown in figures 1 and 2 .

\section{Multivariate Cox proportional hazards model}

A Cox model was made for demographic variables (age, gender, smoking status, ECOG performance status [PS]) and for variables determined as significant for predicting OS or PFS in univariate analysis. We observed significantly better OS for within-normal LDH, albumin, and NLR, as well as a trend relative to $\mathrm{Na}(p=0.077)$. Values of these laboratory parameters outside the established standards were associated with poorer OS. We observed significantly better PFS for ECOG PS, CRP, albumin, and NLR within normal. Values of CRP, albumin, and NLR outside the established standards were associated with poorer PFS. Results are summarized in Table 4. 


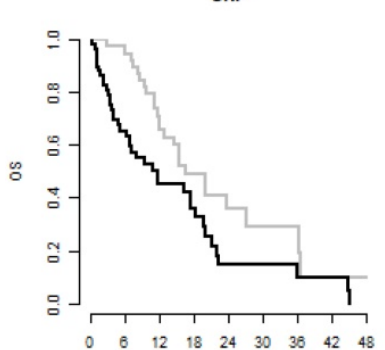

Time (months)

Hemoglobin

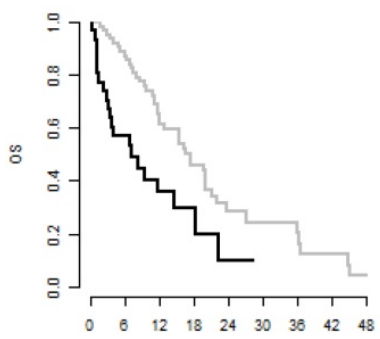

Time (months)
LDH

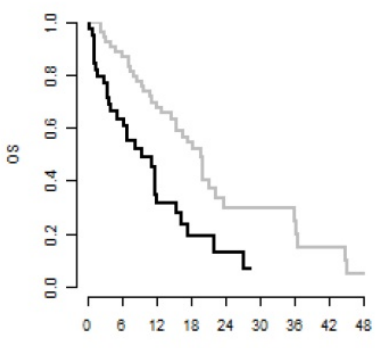

Time (months)

Neutrophils

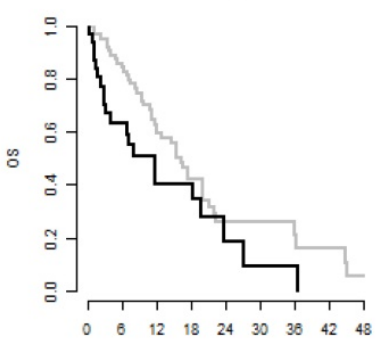

Time (months)

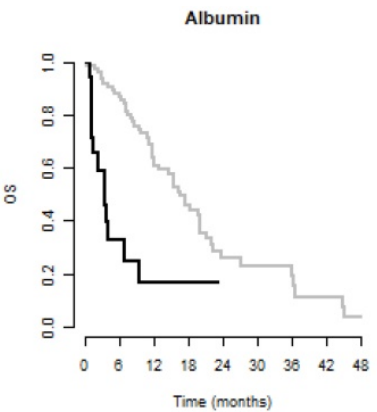

NLR

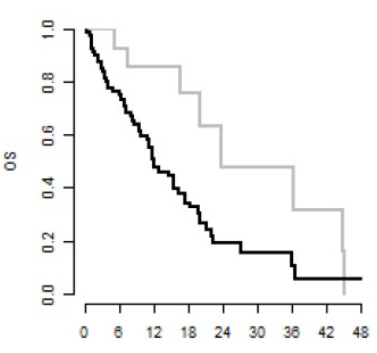

Sodium
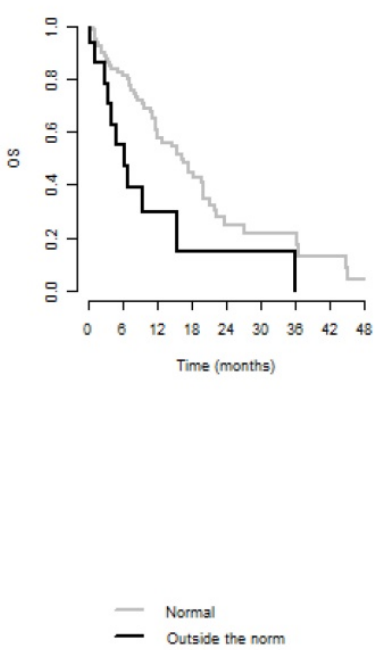

Figure 1. Kaplan-Meier curves of parameters significant for OS
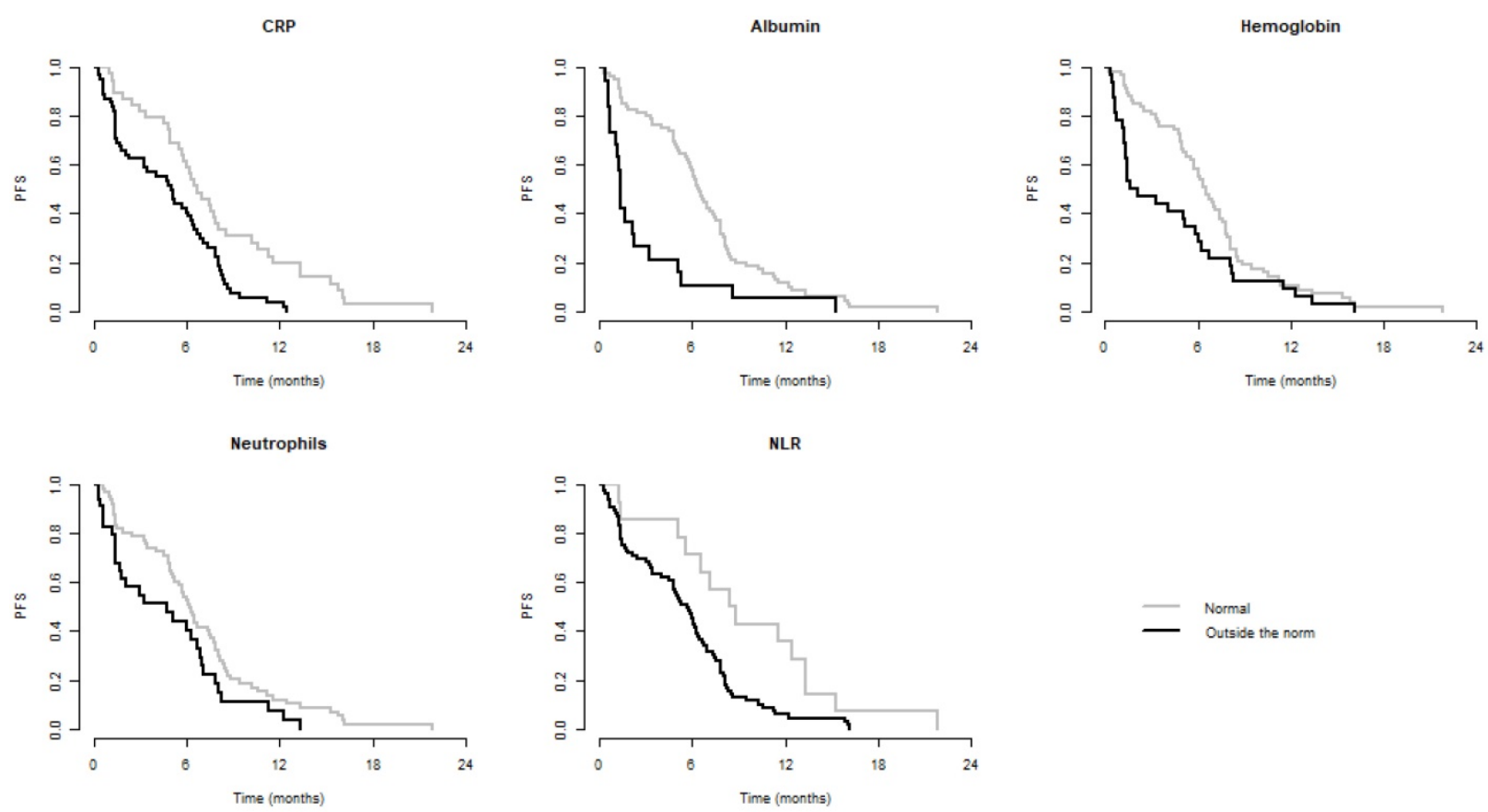

Figure 2. Kaplan-Meier curves of parameters significant for PFS

Table 3. Relationships between laboratory parameters and OS and PFS.

\begin{tabular}{|c|c|c|c|c|c|c|}
\hline \multirow[b]{2}{*}{ Parameter } & \multicolumn{3}{|c|}{ Median PFS (95\% CI), months } & \multicolumn{3}{|c|}{ Median OS (95\% CI), months } \\
\hline & Normal & Outside norm & p-value & Normal & Outside norm & p-value \\
\hline CRP & $6.7(5.8-8.5)$ & $5.1(3.3-6.5)$ & 0.001 & 16.8 (12.9-NR) & $11.7(6.9-19.7)$ & 0.013 \\
\hline $\mathrm{LDH}$ & $6.0(5.0-7.9)$ & $5.2(3.0-6.7)$ & 0.083 & $19.7(15.5-23.9)$ & $9.6(5.1-15.6)$ & $<0.001$ \\
\hline Albumin & $6.5(5.8-7.6)$ & $1.3(1.2-5.1)$ & $<0.001$ & $16.8(12.9-21.2)$ & 3.7 (1.6-NR) & $<0.001$ \\
\hline Sodium & $6.0(5.1-7.6)$ & $4.8(3.0-7.1)$ & 0.055 & $16.3(12.0-20.1)$ & $6.3(4.0-\mathrm{NR})$ & 0.007 \\
\hline Hemoglobin & $6.5(5.7-7.8)$ & $1.9(1.3-6.0)$ & 0.023 & $17.5(12.9-22.1)$ & 7.3 (3.4-NR) & 0.002 \\
\hline Neutrophils & $6.2(5.3-7.8)$ & $4.8(1.7-7.0)$ & 0.040 & $16.3(12.1-21.2)$ & 11.7 (4.1-NR) & 0.050 \\
\hline NLR & $6.9(6.3-8.5)$ & $4.0(1.6-6.0)$ & $<0.001$ & 19.7 (16.3-NR) & $9.4(4.8-15.6)$ & 0.003 \\
\hline
\end{tabular}

CRP: C-reactive protein; LDH: lactate dehydrogenase; NR: not reached; NLR: neutrophil /lymphocyte ratio 
Table 4. Multivariate Cox proportional hazards model for OS and PFS.

\begin{tabular}{|c|c|c|c|c|c|}
\hline & & OS & & PFS & \\
\hline Category & Subcategory & HR (95\% CI) & p-value & HR (95\% CI) & p-value \\
\hline \multirow[t]{2}{*}{ Gender } & Male & Reference & $\mathrm{x}$ & Reference & $\mathrm{x}$ \\
\hline & Female & $1.428(0.742 ; 2.748)$ & 0.287 & $1.329(0.799 ; 2.210)$ & 0.273 \\
\hline Age & & $0.997(0.967 ; 1.027)$ & 0.828 & $0.996(0.969 ; 1.024)$ & 0.775 \\
\hline \multirow[t]{3}{*}{ Smoking status } & Non-smoker & Reference & $\mathrm{x}$ & Reference & $\mathrm{x}$ \\
\hline & Ex-smoker & $0.925(0.398 ; 2.146)$ & 0.856 & $0.825(0.409 ; 1.664)$ & 0.591 \\
\hline & Smoker & $1.231(0.580 ; 2.614)$ & 0.588 & $0.929(0.506 ; 1.707)$ & 0.814 \\
\hline \multirow[t]{3}{*}{ ECOG PS } & 0 & Reference & $\mathrm{x}$ & Reference & $\mathrm{x}$ \\
\hline & 1 & $0.678(0.211 ; 2.180)$ & 0.515 & $0.293(0.094 ; 0.912)$ & 0.034 \\
\hline & 2 & $1.134(0.280 ; 4.596)$ & 0.861 & $0.575(0.152 ; 2.176)$ & 0.415 \\
\hline \multirow[t]{2}{*}{ CRP } & Normal & Reference & $\mathrm{x}$ & Reference & $x$ \\
\hline & Outside norm & $1.337(0.728 ; 2.455)$ & 0.349 & $1.886(1.099 ; 3.237)$ & 0.021 \\
\hline \multirow[t]{2}{*}{$\mathrm{LDH}$} & Normal & Reference & $\mathrm{x}$ & $x$ & \\
\hline & Outside norm & $1.972(1.074 ; 3.622)$ & 0.029 & & \\
\hline \multirow[t]{2}{*}{ Albumin } & Normal & Reference & $\mathrm{x}$ & $\mathrm{x}$ & $\mathrm{x}$ \\
\hline & Outside norm & $3.860(1.845 ; 8.078)$ & $<0.001$ & $3.023(1.681 ; 5.437)$ & $<0.001$ \\
\hline \multirow[t]{2}{*}{ Sodium } & Normal & Reference & $\mathrm{x}$ & $x$ & \\
\hline & Outside norm & $1.980(0.928 ; 4.228)$ & 0.077 & & \\
\hline \multirow[t]{2}{*}{ Hemoglobin } & Normal & Reference & $\mathrm{x}$ & Reference & $\mathrm{x}$ \\
\hline & Outside norm & $1.581(0.836 ; 2.992)$ & 0.159 & $1.282(0.788 ; 2.088)$ & 0.317 \\
\hline \multirow[t]{2}{*}{ NLR } & Normal & Reference & $\mathrm{x}$ & Reference & $\mathrm{x}$ \\
\hline & Outside norm & $1.818(1.007 ; 3.281)$ & 0.047 & $2.273(1.427 ; 3.620)$ & 0.001 \\
\hline
\end{tabular}

CRP: C-reactive protein; ECOG PS: Eastern Cooperative Oncology Group performance status; LDH: lactate dehydrogenase; NLR: neutrophil/lymphocyte ratio

\section{Discussion}

The data from the present retrospective analysis indicate possible prognostic and theoretically predictive value for some of those laboratory parameters examined. To the best of our knowledge, this work is the first in the English-language literature (within the PubMed Database) to date to examine the relationships between $\mathrm{CRP}, \mathrm{Hb}$, and $\mathrm{Na}$ and the efficacy of bevacizumab treatment in NSCLC. Our work is also unique in relation to the complexity of the baseline aspects of laboratory marker values with respect to the effect of bevacizumab treatment in NSCLC.

LDH and Na showed possible prognostic value in NSCLC patients treated with bevacizumab plus chemotherapy. Adverse prognostic effects of hyponatremia on patients with NSCLC have been demonstrated in other studies concerning NSCLC and other treatment combinations [8, 19]. We found no other studies dealing with the relationship between $\mathrm{Na}$ and bevacizumab treatment in NSCLC. With regard to $\mathrm{LDH}$, the literature is much richer. A meta-analysis of the relationship between LDH and bevacizumab treatment in colorectal cancer (CRC) showed significant relationships for both PFS and OS [20]. The effect on the response to treatment has not been proven, however, so a question remains whether the relationship with $\mathrm{LDH}$ is predictive or only prognostic in CRC. In our study of NSCLC, we demonstrated only a prognostic value. The prognostic value of LDH in NSCLC patients treated with bevacizumab was demonstrated also by the study of
Honag et al. [21]. Li et al. then demonstrated a relationship between LDH and both OS and PFS in a univariate model, but this was not confirmed in Cox's multidimensional model [22]. On the contrary, they point to the possible importance for the development of LDH levels during treatment with bevacizumab. This was suggested, too, by a study from Silvestis et al. in relation to CRC [23]. This can be supported also by preclinical data suggesting a relationship between $\mathrm{LDH}$, angiogenesis, and VEGF [24, 25]. Because serum level of LDH was shown to be an indirect factor indicative for hypoxia in tumor tissues with large tumor burden, it seems plausible that the improvement of hypoxia in the tumor microenvironment by bevacizumab could be reflected in a decrease of LDH serum level [22]. It was not, however, a goal of our work to examine this. In our opinion, the baseline LDH can only be considered as a prognostic marker. For it to have possible predictive value, it would be necessary to verify the relationship to its changes during BEV treatment. Finally, different LDH isoforms might have different functions in this setting [26], and this is not normally detected in routine laboratory samples. This can complicate the potential for $\mathrm{LDH}$ to be used in routine clinical practice.

The parameters associated with the inflammatory tumor microenvironment [10] are, in our view, more promising predictive markers for $\mathrm{BEV}$ treatment in NSCLC. VEGF, a target of BEV, is a soluble dimeric protein with multiple bioregulative activities that is mainly released in hypoxic and inflammatory conditions [27]. VEGF bioregulative activity is very complex, and it also involves the 
anticancer immune system [27]. Several studies in relation to $\mathrm{CRC}$, breast cancer, and renal cell cancer have demonstrated that bevacizumab is most advantageous in extending PFS and/or OS in patients who presented a lower systemic inflammatory profile prior to beginning the treatment [12, 13, 15, 28-31]. These studies proved the positive relationship between unincreased values of Neu or NLR and CRP, as well as of higher $\mathrm{Hb}$ values, and better results of BEV treatment [12, 13, 15, 28-31]. An effect of inflammation on poorer treatment outcomes has been demonstrated also with other treatment modalities in NSCLC [9-11]. We also found two studies directly addressing the relationship of selected parameters associated with inflammatory response to BEV treatment in NSCLC [21, 32]. Hoang et al. demonstrate in their study an adverse effect of hypoalbuminemia on OS and PFS [21]. Botta et al. then point to a possible effect from high numbers of circulating neutrophils and monocytes as well as high NLR on the results of bevacizumab treatment [32]. Neither of these studies, however, addressed the relationship to ORR. They also did not consider the usefulness of CRP and $\mathrm{Hb}$ as important parameters of inflammation [10]. In this regard, our work is the most comprehensive of its kind in predicting BEV therapy. We demonstrated the relationship between CRP, albumin, $\mathrm{Hb}, \mathrm{Neu}$, and NLR on ORR and also that of CRP, albumin, and NLR on PFS in a multivariate Cox model. Only $\mathrm{Hb}$ did not confirm its relationship to FPS from the univariate model in the Cox model. Although decline in $\mathrm{Hb}$ during ongoing inflammation is known, its decrease may be associated with other effects that may have been reflected in the Cox model $[33,34]$.

The present study has several limitations. First, this was a retrospective study that could be biased with regard to patient selection for BEV plus chemotherapy regimen. Secondly, PFS was not confirmed by an independent board. Finally, we examined a relatively limited number of patients and therefore some analyses may lack sufficient statistical power. The present report should thus be regarded as exploratory and the results should be verified in a larger, prospective study. Determining the optimal cutoff for NLR (which has varied from 2.5 to 5 in studies analyzing the possible relationship of NLR to the outcome of bevacizumab treatment) may also be problematic for subsequent validation $[12,28,29,32$, $35,36]$.

\section{Conclusions}

$\mathrm{LDH}$ and $\mathrm{Na}$ appear to be possible prognostic markers for bevacizumab treatment in combination with chemotherapy in NSCLC. The parameters associated with the inflammatory response (CRP, $\mathrm{NLR}$, albumin, and possibly $\mathrm{Hb}$ ), then, appear to be promising predictive markers for this treatment combination. It would be appropriate to verify their use (probably and preferably in the form of a proinflammatory index) in a prospective study.

\section{Abbreviations}

BEV: bevacizumab; $\mathrm{CP}$ : carboplatin and paclitaxel; CRC: colorectal cancer; CRP: C-reactive protein; CT: computed tomography; ECOG: Eastern Cooperative Oncology Group; ECOG PS: ECOG performance status; $\mathrm{Hb}$ : hemoglobin; $\mathrm{LDH}$ : lactate dehydrogenase; Neu: neutrophils; NLR: neutrophil/lymphocyte ratio; NSCLC: non-small cell lung cancer; ORR: overall response rate; OS: overall survival; PFS: progression-free survival; RECIST: Response Evaluation Criteria in Solid Tumors; VEGF: vascular endothelial growth factor.

\section{Acknowledgements}

This study was supported by the grant of the Ministry of Health of the Czech Republic - Conceptual Development of Research Organization (Faculty Hospital in Pilsen - FNPl, 00669806). Publication was also supported by the Czech Pneumological and Phthisiological Society. The authors thank Gale A. Kirking, Editor-in-Chief at English Editorial Services, s.r.o., for the careful, professional editing of our text.

\section{Author Contributions}

MS and JB conceived the presented idea. MS, JB, $\mathrm{GK}, \mathrm{MB}, \mathrm{ZT}, \mathrm{MP}$, and JV conceived and planned the experiments and collected the data. $\mathrm{KH}$ and $\mathrm{MB}$ analyzed the data. MS wrote the article with support from JB. MP helped supervise the project.

\section{Competing interests}

In connection with this article, the authors declare having provided consulting services to Roche in the past.

\section{References}

1. Hong S, Tan M, Wang S, et al. Efficacy and safety of angiogenesis inhibitors in advanced non-small cell lung cancer: a systematic review and meta-analysis. J Cancer Res Clin Oncol. 2015; 141(5): 909-21.

2. Sandler A, Gray R, Perry MC, et al. Paclitaxel-carboplatin alone or with bevacizumab for non-small-cell lung cancer. N Engl J Med. 2006; 355(24): 2542-50.

3. Ramalingam SS, Belani CP. Antiangiogenic agents in the treatment of nonsmall cell lung cancer: reality and hope. Curr Opin Oncol. 2010; 22(2): 79-85

4. Dowlati A, Gray R, Sandler AB, et al. Cell adhesion molecules, vascular endothelial growth factor, and basic fibroblast growth factor in patients with non-small cell lung cancer treated with chemotherapy with or without bevacizumab--an Eastern Cooperative Oncology Group Study. Clin Cancer Res. 2008; 14(5): 1407-12.

5. Evans T. Utility of hypertension as a surrogate marker for efficacy of antiangiogenic therapy in NSCLC. Anticancer Res. 2012; 32(11): 4629-38.

6. $\mathrm{Ng} \mathrm{QS}$, Goh V. Angiogenesis in non-small cell lung cancer: imaging with perfusion computed tomography. J Thorac Imaging. 2010; 25(2): 142-50, 2010. 
7. Salgia R. Prognostic significance of angiogenesis and angiogenic growth factors in nonsmall cell lung cancer. Cancer. 2011; 117(17): 3889-99.

8. Svaton M, Fiala O, Pesek M, et al. Predictive and prognostic significance of sodium levels in patients with NSCLC treated by erlotinib. Anticancer Res. 2014; 34(12): 7461-5

9. Fiala O, Pesek M, Finek J, et al. High serum level of C-reactive protein is associated with worse outcome of patients with advanced-stage NSCLC treated with erlotinib. Tumour Biol. 2015; 36(12): 9215-22.

10. Svaton M, Zemanova M, Skrickova J, et al. Chronic Inflammation as a Potential Predictive Factor of Nivolumab Therapy in Non-small Cell Lung Cancer. Anticancer Res. 2018; 38(12): 6771-6782.

11. Fiala O, Hosek P, Pesek M, et al. Prognostic role of serum C-reactive protein in patients with advanced-stage NSCLC treated with pemetrexed. Neoplasma. 2017; 64(4): 605-610.

12. Artaç $M$, Uysal $M$, Karaağaç $M$, et al. Prognostic Impact of Neutrophil/Lymphocyte Ratio, Platelet Count, CRP, and Albumin Levels in Metastatic Colorectal Cancer Patients Treated with FOLFIRI-Bevacizumab. J Gastrointest Cancer. 2017; 48(2): 176-180.

13. Miyagawa Y, Yanai A, Yanagawa T, et al. Baseline neutrophil-to-lymphocyte ratio and c-reactive protein predict efficacy of treatment with bevacizumab plus paclitaxel for locally advanced or metastatic breast cancer. Oncotarget. 2020; 11(1): 86-98.

14. Nixon AB, Pang H, Starr MD, et al. Prognostic and predictive blood-based biomarkers in patients with advanced pancreatic cancer: results from CALGB80303 (Alliance). Clin Cancer Res. 2013; 19(24): 6957-6966.

15. Heng DY, Xie W, Regan MM, et al. Prognostic factors for overall survival in patients with metastatic renal cell carcinoma treated with vascular endothelial growth factor-targeted agents: results from a large, multicenter study. J Clin Oncol. 2009; 27(34): 5794-5799.

16. Bertaut A, Truntzer C, Madkouri R, et al. Blood baseline neutrophil count predicts bevacizumab efficacy in glioblastoma. Oncotarget. 2016; 7(43): 70948-70958.

17. Farolfi A, Petrone M, Scarpi E, et al. Inflammatory Indexes as Prognostic and Predictive Factors in Ovarian Cancer Treated with Chemotherapy Alone or Together with Bevacizumab. A Multicenter, Retrospective Analysis by the MITO Group (MITO 24). Target Oncol. 2018; 13(4): 469-479.

18. Eisenhauer EA, Therasse $\mathrm{P}$, Bogaerts J, et al. New response evaluation criteria in solid tumours: revised RECIST guideline (version 1.1). Eur J Cancer. 2009; 45(2): 228-47.

19. Zarzecka M, Kubicki P, Kozielski J. Hyponatraemia - evaluation of prevalence in patients hospitalized in the Pulmonary Department and prognostic significance in lung cancer patients. Pneumonol Alergol Pol. 2014; 82(1): 18-24.

20. Feng W, Wang Y, Zhu X. Baseline serum lactate dehydrogenase level predicts survival benefit in patients with metastatic colorectal cancer receiving bevacizumab as first-line chemotherapy: a systematic review and meta-analysis of 7 studies and 1,219 patients. Ann Transl Med. 2019; 7(7): 133.

21. Hoang T, Dahlberg SE, Sandler AB, et al. Prognostic models to predict survival in non-small-cell lung cancer patients treated with first-line paclitaxel and carboplatin with or without bevacizumab. J Thorac Oncol. 2012; 7(9): 1361-1368.

22. Li B, Li C, Guo M, et al. Predictive value of $\mathrm{LDH}$ kinetics in bevacizumab treatment and survival of patients with advanced NSCLC. Onco Targets Ther. 2018; 11: 6287-6294.

23. Silvestris N, Scartozzi M, Graziano G, et al. Basal and bevacizumab-based therapy-induced changes of lactate dehydrogenases and fibrinogen levels and clinical outcome of previously untreated metastatic colorectal cancer patients: a multicentric retrospective analysis. Expert Opin Biol Ther. 2015; 15(2): 155-162.

24. Harris AL. Hypoxia--a key regulatory factor in tumour growth. Nat Rev Cancer. 2002; 2(1): 38-47.

25. Azuma M, Shi M, Danenberg KD, et al. Serum lactate dehydrogenase levels and glycolysis significantly correlate with tumor VEGFA and VEGFR expression in metastatic CRC patients. Pharmacogenomics. 2007; 8(12): 1705-13.

26. Giampieri R, Puzzoni M, Daniele B, et al. First-line FOLFIRI and bevacizumab in patients with advanced colorectal cancer prospectively stratified according to serum LDH: final results of the GISCAD (Italian Group for the Study of Digestive Tract Cancers) CENTRAL (ColorEctalavastiNTRiAlLdh) trial. Br J Cancer. 2017; 117(8): 1099-1104.

27. Martino EC, Misso G, Pastina $P$, et al. Immune-modulating effects of bevacizumab in metastatic non-small-cell lung cancer patients. Cell Death Discov. 2016; 2: 16025.

28. Casadei-Gardini A, Scarpi E, Ulivi $\mathrm{P}$, et al. Prognostic role of a new inflammatory index with neutrophil-to-lymphocyte ratio and lactate dehydrogenase (CII: Colon Inflammatory Index) in patients with metastatic colorectal cancer: results from the randomized Italian Trial in Advanced Colorectal Cancer (ITACa) study. Cancer Manag Res. 2019; 11: 4357-4369.

29. Clarke SJ, Burge M, Feeney K, et al. The prognostic role of inflammatory markers in patients with metastatic colorectal cancer treated with bevacizumab: A translational study [ASCENT]. PLoS One. 2020; 15(3): e0229900.

30. Cetin B, Kaplan MA, Berk V, et al. Prognostic factors for overall survival in patients with metastatic colorectal carcinoma treated with vascular endothelial growth factor-targeting agents. Asian Pac J Cancer Prev. 2012; 13(3): 1059-1063.
31. Harshman LC, Kuo CJ, Wong BY, et al. Increased hemoglobin associated with VEGF inhibitors in advanced renal cell carcinoma. Cancer Invest. 2009; 27(8): 851-856.

32. Botta C, Barbieri V, Ciliberto D, et al. Systemic inflammatory status at baseline predicts bevacizumab benefit in advanced non-small cell lung cancer patients. Cancer Biol Ther. 2013; 14(6): 469-475.

33. Fraenkel PG. Anemia of Inflammation: A Review. Med Clin North Am. 2017; 101(2): 285-296.

34. Macciò A, Madeddu C, Gramignano G, et al. The role of inflammation, iron, and nutritional status in cancer-related anemia: results of a large, prospective, observational study. Haematologica. 2015; 100(1): 124-32.

35. Dogan E, Bozkurt O, Sakalar T, et al. Impact of neutrophil-lymphocyte and platelet-lymphocyte ratio on antiEGFR and bevacizumab efficacy in metastatic colorectal cancer. J BUON. 2019; 24(5): 1861-1869.

36. Dirican A, Varol U, Kucukzeybek Y, et al. Treatment of metastatic colorectal cancer with or without bevacizumab: can the neutrophil/lymphocyte ratio predict the efficiency of bevacizumab?. Asian Pac J Cancer Prev. 2014; 15(12): $4781-4786$. 